\title{
Impact of multimorbidity: acute morbidity, area of residency and use of health services across the life span in a region of south Europe
}

Quintí Foguet-Boreu ${ }^{1,2,3^{*}}$, Concepció Violan ${ }^{1,2}$, Albert Roso-Llorach ${ }^{1,2}$, Teresa Rodriguez-Blanco ${ }^{1,2}$, Mariona Pons-Vigués ${ }^{1,2}$, Miguel A Muñoz-Pérez ${ }^{1,2}$, Enriqueta Pujol-Ribera ${ }^{1,2}$ and Jose M Valderas ${ }^{4}$

\begin{abstract}
Background: Concurrent diseases, multiple pathologies and multimorbidity patterns are topics of increased interest as the world's population ages. To explore the impact of multimorbidity on affected patients and the consequences for health services, we designed a study to describe multimorbidity by sex and life-stage in a large population sample and to assess the association with acute morbidity, area of residency and use of health services.

Methods: A cross-sectional study was conducted in Catalonia (Spain). Participants were 1,749,710 patients aged $19+$ years (251 primary care teams). Primary outcome: Multimorbidity ( $\geq 2$ chronic diseases). Secondary outcome: Number of new events of each acute disease. Other variables: number of acute diseases per patient, sex, age group (19-24, 25-44, 45-64, 65-79, and 80+ years), urban/rural residence, and number of visits during 2010.

Results: Multimorbidity was present in $46.8 \%$ (95\% Cl, 46.7\%-46.8\%) of the sample, and increased as age increased, being higher in women and in rural areas. The most prevalent pair of chronic diseases was hypertension and lipid disorders in patients older than 45 years. Infections (mainly upper respiratory infection) were the most common acute diagnoses. In women, the highest significant RR of multimorbidity vs. non-multimorbidity was found for teeth/gum disease (aged 19-24) and acute upper respiratory infection. In men, this RR was only positive and significant for teeth/gum disease (aged 65-79). The adjusted analysis showed a strongly positive association with multimorbidity for the oldest women (80+ years) with acute diseases and women aged 65-79 with 3 or more acute diseases, compared to patients with no acute diseases (OR ranged from 1.16 to $1.99, \mathrm{p}<0.001$ ). Living in a rural area was significantly associated with lower probability of having multimorbidity. The odds of multimorbidity increased sharply as the number of visits increased, reaching the highest probability in those aged 65-79 years.

Conclusions: Multimorbidity is related to greater use of health care services and higher incidence of acute diseases, increasing the burden on primary care services. The differences related to sex and life-stage observed for multimorbidity and acute diseases suggest that further research on multimorbidity should be stratified according to these factors.
\end{abstract}

Keywords: Multimorbidity, Chronic disease, Acute disease, Life-stage

\footnotetext{
*Correspondence: qfoguet@idiapjgol.org

'Institut Universitari d'Investigació en Atenció Primària Jordi Gol (IDIAP Jordi

Gol), Gran Via Corts Catalanes, 587 àtic, 08007 Barcelona, Spain

${ }^{2}$ Universitat Autònoma de Barcelona, Plaza Cívica, Campus de la UAB, 08193

Bellaterra, Cerdanyola del Vallès, Spain

Full list of author information is available at the end of the article
}

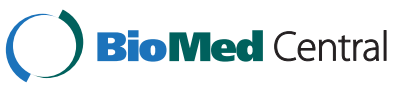

(C) 2014 Foguet-Boreu et al.; licensee BioMed Central Ltd. This is an Open Access article distributed under the terms of the Creative Commons Attribution License (http://creativecommons.org/licenses/by/2.0), which permits unrestricted use, distribution, and reproduction in any medium, provided the original work is properly credited. The Creative Commons Public Domain Dedication waiver (http://creativecommons.org/publicdomain/zero/1.0/) applies to the data made available in this article, unless otherwise stated. 


\section{Background}

Concurrent diseases, multiple pathologies and multimorbidity patterns are topics of increased interest as the world's population ages [1]. Multimorbidity is the coexistence of two or more chronic health problems in the same person at one point in time [2], and multimorbidity patterns are any combination of chronic diseases [3]. Both considerations have important consequences for the individual and for health services [4]. Multimorbidity is a challenge for industrialized countries and can jeopardize the viability of national health systems.

Traditionally, the construct of multimorbidity has been inherently associated with persistent or chronic disease. Methods to measure multimorbidity include disease scores, case-mix systems, indexes and disease counts, the latter being the common method [5]. Far too little attention has been paid to the use of health services and the role of urban or rural residency in patients with multimorbidity [6,7]. Furthermore, the literature lacks comparisons by sex of acute morbidity in patients with multimorbidity in a large population sample $[8,9]$.

The classification of acute and chronic disease remains controversial. Acute disease is characterized by a single or repeated episode of relatively rapid onset and short duration with a recovery to previous stage of activity [10]. Nevertheless, some diseases fall into a grey area. Knowledge of specific acute diseases that may occur more frequently than expected and of the underlying vulnerabilities [11] could help to focus attention on the patients with multimorbidity rather than emphasizing the diseases.

To explore the impact of multimorbidity on affected patients and the consequences for health services, we designed a study to describe multimorbidity by sex and life-stage in a large population sample and to assess the association with acute morbidity, area of residency and use of health services.

\section{Methods}

Data source and study population

Cross-sectional study of adults resident in Catalonia, a Mediterranean region of southern European with 7,434,632 inhabitants (2010 census), 16\% of the population of Spain. In Catalonia, 358 primary health care teams (PHCT) comprised of doctors, nurses, social workers and support staff are assigned by geographical area and responsible for the health care of the population in their areas. The Catalan Health Institute (CHI) manages 274 PHCT (76.5\%), serving a population of 4,859,725 adults; the remaining PHCT are managed by other providers. Doctors and nurses systematically use electronic health records (EHR) to record diagnoses, prescriptions and other clinical, patient management and administrative activities. The CHI Information System for the Development of Research in Primary Care (SIDIAP) [12] compiles coded clinical information from the EHR system. A subset of SIDIAP records meeting the highest quality criteria for clinical data (SIDIAP-Q) includes $40 \%$ of the SIDIAP population (1,936,443 patients), attended by the 1,319 general practitioners (GP) whose data recording scored highest in a validated comparison process. The sample is representative of the general Catalan population in terms of geography, age and sex distributions, according to the official 2010 census [13].

A sample of 1,749,710 patients aged 19 years or older, assigned to 251 PHCT during the period of study (1 January- 31 December 2010), was selected from the SIDIAP-Q database.

\section{Coding of diseases}

International Classification of Diseases (ICD-10) codes were mapped to the International Classification of Primary Care (ICPC-2e-v.4.2, available at: http://www.kith.no/templates/ kith_WebPage__1111.aspx). R codes (symptoms, signs and abnormal clinical and laboratory findings, not elsewhere classified) and Z codes (factors influencing health status and contact with health services) were excluded, resulting in 686 included codes. Each diagnosis was then classified using O'Halloran criteria for chronic disease [14]. We included all 146 diagnoses considered as chronic diseases by O'Halloran criteria: (i) have a duration that has lasted, or is expected to last, at least 6 months; (ii) have a pattern of recurrence or deterioration; (iii) have a poor prognosis and (iv) produce consequences, or sequelae, that have an impact on the individual's quality of life $[14,15]$. Any disease not meeting the O'Halloran criteria was considered an acute disease.

All results were described with ICPC-2 codes. Diseases were classified as acute if diagnosed during the study period and chronic if recorded as such in EHR as of 31 December 2010.

\section{Outcomes and variables}

The main outcome was multimorbidity, defined as the coexistence of 2 or more chronic diseases. Secondary outcome was the number of new events of each acute disease. Other variables recorded for each patient were the following: number of all acute diseases $(0,1,2,>=3)$, sex (male, female), age (young adult, 19 to 24; adult, 2544; older adult, 45-64; elderly, 65-79; and oldest adult, $80+)$, number of visits during the study period $(0,1-2$, $3-5,6-10, \geq 11$ ), and area of residence (rural if $<10,000$ inhabitants and/or population density $<150$ people $/ \mathrm{km}^{2}$, otherwise urban) [14]. Number of all acute diseases (or 0 diseases) and visits (or 0 visits) were categorized as quartiles of the study population. Number of visits was used as a proxy of use of health services and included visits recorded in EHR by GP, nurses or social workers, either at the primary care centre or as home health care. 


\section{Statistical analysis}

Analysis was stratified by sex and age group. Descriptive statistics were used to summarize overall information. Categorical variables were expressed as frequencies (percentage) and continuous as mean (Standard deviation, SD) or median (interquartile range, IQR).

Cumulative incidence of acute morbidity events was calculated as the number of new acute events during the study period divided by the at-risk population in the sample (e.g., if a patient had bronchitis twice in the oneyear study period, the total number of events accounted for was 2). We took into account the five acute diseases with the highest cumulative incidence within each stratum. Risk ratios (RR) of multimorbidity vs. non-multimorbidity were calculated for the number of events for each acute disease, using Poisson, negative binomial (if overdispersion was present) or zero inflated (when data had an excess of zero counts) equations, as appropriate. All models were adjusted for number of visits and area of residency.

To determine the most prevalent multimorbidity patterns, all possible combinations of any two chronic diseases and their frequencies were calculated. Observed (O) and expected (E) prevalence of those two chronic diseases with each acute disease was then computed. Expected cooccurrence of diseases was obtained as the product of these prevalences, assuming statistical independence of the diseases concerned. The overlapping of those combinations that presented the highest $\mathrm{O} / \mathrm{E}$ ratio was reported.

Logistic regression was used to assess the association between multimorbidity and the variables listed above.

All statistical tests were two-sided at the 5\% significance level. The analyses were performed using SPSS for Windows, version 18 (SPSS Inc., Chicago, IL, USA), Stata/ SE version 11 for Windows (Stata Corp. LP, College Station, TX, USA) and R version 2.15.2 (R Foundation for Statistical Computing, Vienna, Austria).

\section{Ethical considerations}

The study protocol was approved by the Committee on the Ethics of Clinical Research, Institut Universitari d'Investigació en Atenció Primària (IDIAP) Jordi Gol (Protocol No: P12/28). All data were anonymized and the confidentiality of EHR was respected at all times in accordance with international law.

\section{Results}

We included $1,749,710$ patients; mean age was 47.4 years (SD: 17.8$), 50.7 \%$ were female and $16 \%$ lived in rural areas. Multimorbidity ( $\geq 2$ diseases) was present in $46.8 \%$ (95\% CI, $46.7 \%-46.8 \%$ ) of the sample, being higher in female (52.3\%) than in male $(41.1 \%)$ and in rural areas $(47.6 \%)$ than in urban areas (46.6\%).

The prevalence of the most common chronic diseases differed by sex below 45: anxiety disorder/anxiety (women aged 19-44); acne (men aged 19-24) and lipid disorder (men aged 25-44). After 45 both sex groups first chronic disease was lipid disorder in 45-64 and uncomplicated hypertension in 65-80+ (Table 1). Upper respiratory infection acute is the most incident acute disease in all age groups (except in $80+$ ).

Multimorbidity prevalence increased as age increased, being higher in female (ranged from 19.0\% to 92.1\%) than male (ranged from $12.9 \%$ to $92.0 \%$ ). In patients with multimorbidity, the number of acute diseases was higher in female than male and decreased as age increased, except in male older than 65 . In addition, the number of visits increased as age increased, and was higher for female than male in all age groups except $80+$ (Table 2).

Patients with multimorbidity had a higher incidence of acute diseases and number of visits in all age strata than non-multimorbidity patients; in both cases, the incidence was higher for female than male (Table 2). Overall, the median (IQR) of number of visits was 8(4-14) in patients with multimorbidity vs. $1(0-4)$ in the non-multimorbidity group.

The two most prevalent combinations of two chronic diseases were hypertension and lipid disorders in patients older than 45 years. The only acute disease that appeared in both sexes was "bursitis/tendinitis/synovitis NOS" in the oldest age group $(80+)$. In the other age groups, the acute health disease varied by sex (Figure 1).

The five acute diseases with the highest cumulative incidence were similar by sex in any age group. Infections were the most common diagnosis. Cystitis/urinary infection was present among the five most prevalent acute conditions only in women and in the oldest men. In women, the highest significant RR of multimorbidity vs. non-multimorbidity was found for teeth/gum disease (aged 19-24) and upper respiratory infection, acute (80+). In men, this RR was only positive and significant for teeth/ gum disease (aged 65-79) (Table 3).

The adjusted analysis of factors associated with multimorbidity showed that the oldest patients $(80+$ years) with acute diseases and women aged 65-79 with 3 or more acute diseases were more likely to have multimorbidity than patients with no acute diseases. This positive association was only significant in women. Living in a rural area was significantly associated with lower probability of having multimorbidity. Patients who visited a GP more often were more likely than those without visits to have multimorbidity, reaching the highest probability in those aged 65-79 years (Table 4).

\section{Discussion}

\section{Statement of principal findings}

Almost half of the study population had multimorbidity, with infections (mainly acute upper respiratory infection) the most common acute disease in both sexes and all age 
Table 1 Five highest cumulative incidence of acute and prevalence of chronic diseases by sex and age groups

\begin{tabular}{|c|c|c|c|c|c|c|c|c|c|c|c|c|}
\hline \multicolumn{7}{|l|}{ Female } & \multicolumn{6}{|l|}{ Male } \\
\hline $\begin{array}{l}\text { Age } \\
\text { groups }\end{array}$ & ICPC & Chronic diseases & $\begin{array}{l}\text { Prevalence } \\
(\%, \mathrm{Cl})\end{array}$ & ICPC & Acute diseases & $\begin{array}{l}\text { Cumulative } \\
\text { incidence }(\%, \mathrm{Cl})\end{array}$ & ICPC & Chronic diseases & $\begin{array}{l}\text { Prevalence } \\
(\%, \mathrm{Cl})\end{array}$ & ICPC & Acute diseases & $\begin{array}{l}\text { Cumulative } \\
\text { incidence }(\%, \mathrm{Cl})\end{array}$ \\
\hline \multirow[t]{5}{*}{$19-24$} & P74 & $\begin{array}{l}\text { Anxiety disorder/ } \\
\text { anxiety state }\end{array}$ & $8.4(8.2-8.7)$ & R74 & $\begin{array}{l}\text { Upper respiratory } \\
\text { infection acute }\end{array}$ & $9.1(8.9-9.3)$ & S96 & Acne & $7.7(7.5-7.9)$ & R74 & $\begin{array}{l}\text { Upper respiratory } \\
\text { infection acute }\end{array}$ & $6.9(6.7-7.1)$ \\
\hline & S96 & Acne & $7.8(7.6-8.0)$ & R76 & Tonsillitis acute & $4.4(4.3-4.6)$ & R96 & Asthma & $6.0(5.8-6.2)$ & R76 & Tonsillitis acute & $3.4(3.3-3.6)$ \\
\hline & R96 & Asthma & $5.4(5.2-5.6)$ & U71 & $\begin{array}{l}\text { Cystitis/urinary } \\
\text { infection other }\end{array}$ & $4.1(4.0-4.3)$ & P74 & $\begin{array}{l}\text { Anxiety disorder/ } \\
\text { anxiety state }\end{array}$ & $3.9(3.7-4.0)$ & D73 & $\begin{array}{l}\text { Gastroenteritis } \\
\text { presumed infection }\end{array}$ & $2.9(2.8-3.0)$ \\
\hline & T82 & Obesity & $5.0(4.8-5.1)$ & D82 & Teeth/gum disease & $3.7(3.5-3.8)$ & T82 & Obesity & $3.4(3.3-3.6)$ & D82 & Teeth/gum disease & $2.5(2.4-2.7)$ \\
\hline & L85 & $\begin{array}{l}\text { Acquired deformity } \\
\text { of spine }\end{array}$ & $4.6(4.4-4.8)$ & D73 & $\begin{array}{l}\text { Gastroenteritis } \\
\text { presumed infection }\end{array}$ & $3.6(3.5-3.8)$ & L85 & $\begin{array}{l}\text { Acquired deformity } \\
\text { of spine }\end{array}$ & $3.1(3.0-3.2)$ & S16 & Bruise/contusion & $2.3(2.2-2.4)$ \\
\hline \multirow[t]{5}{*}{$25-44$} & P74 & $\begin{array}{l}\text { Anxiety disorder/ } \\
\text { anxiety state }\end{array}$ & $12.2(12.1-12.3)$ & R74 & $\begin{array}{l}\text { Upper respiratory } \\
\text { infection acute }\end{array}$ & $7.8(7.7-7.9)$ & T93 & Lipid disorder & $7.3(7.2-7.4)$ & R74 & $\begin{array}{l}\text { Upper respiratory } \\
\text { infection acute }\end{array}$ & $5.9(5.8-5.9)$ \\
\hline & P76 & Depressive disorder & $8.8(8.7-8.9)$ & L03 & $\begin{array}{l}\text { Low back symptom/ } \\
\text { complaint }\end{array}$ & $3.4(3.3-3.5)$ & P74 & $\begin{array}{l}\text { Anxiety disorder/ } \\
\text { anxiety state }\end{array}$ & $6.5(6.4-6.6)$ & D73 & $\begin{array}{l}\text { Gastroenteritis } \\
\text { presumed infection }\end{array}$ & $2.4(2.4-2.5)$ \\
\hline & T82 & Obesity & $6.8(6.7-6.9)$ & U71 & $\begin{array}{l}\text { Cystitis/urinary } \\
\text { infection other }\end{array}$ & $2.9(2.8-2.9)$ & T82 & Obesity & $4.4(4.4-4.5)$ & L03 & $\begin{array}{l}\text { Low back symptom/ } \\
\text { complaint }\end{array}$ & $2.4(2.3-2.4)$ \\
\hline & T93 & Lipid disorder & $5.0(4.9-5.0)$ & D73 & $\begin{array}{l}\text { Gastroenteritis } \\
\text { presumed infection }\end{array}$ & $2.8(2.8-2.9)$ & P76 & Depressive disorder & $3.7(3.7-3.8)$ & D82 & Teeth/gum disease & $2.1(2.1-2.2)$ \\
\hline & N89 & Migraine & $4.9(4.8-4.9)$ & R76 & Tonsillitis acute & $2.6(2.6-2.7)$ & L86 & $\begin{array}{l}\text { Back syndrome with } \\
\text { radiating pain }\end{array}$ & $3.5(3.4-3.5)$ & R76 & Tonsillitis acute & $1.8(1.8-1.9)$ \\
\hline \multirow[t]{5}{*}{$45-64$} & T93 & Lipid disorder & $28.4(28.2-28.5)$ & R74 & $\begin{array}{l}\text { Upper respiratory } \\
\text { infection acute }\end{array}$ & $7.0(6.9-7.1)$ & T93 & Lipid disorder & $29.9(29.7-30.1)$ & R74 & $\begin{array}{l}\text { Upper respiratory } \\
\text { infection acute }\end{array}$ & $4.8(4.7-4.9)$ \\
\hline & K86 & $\begin{array}{l}\text { Hypertension } \\
\text { uncomplicated }\end{array}$ & $21.2(21.1-21.4)$ & L03 & $\begin{array}{l}\text { Low back symptom/ } \\
\text { complaint }\end{array}$ & $3.2(3.1-3.3)$ & K86 & $\begin{array}{l}\text { Hypertension } \\
\text { uncomplicated }\end{array}$ & $24.6(24.4-24.7)$ & L03 & $\begin{array}{l}\text { Low back symptom/ } \\
\text { complaint }\end{array}$ & $2.6(2.5-2.7)$ \\
\hline & P76 & Depressive disorder & $18.9(18.8-19.1)$ & U71 & $\begin{array}{l}\text { Cystitis/urinary } \\
\text { infection other }\end{array}$ & $2.9(2.8-3.0)$ & T82 & Obesity & $10.9(10.8-11.0)$ & R78 & $\begin{array}{l}\text { Acute bronchitis/ } \\
\text { bronchiolitis }\end{array}$ & $2.0(2.0-2.1)$ \\
\hline & T82 & Obesity & $15.7(15.6-15.9)$ & R78 & $\begin{array}{l}\text { Acute bronchitis/ } \\
\text { bronchiolitis }\end{array}$ & $2.8(2.7-2.9)$ & T90 & $\begin{array}{l}\text { Diabetes non-insulin } \\
\text { dependent }\end{array}$ & $10.3(10.2-10.5)$ & D82 & Teeth/gum disease & $2.0(2.0-2.1)$ \\
\hline & P74 & $\begin{array}{l}\text { Anxiety disorder/ } \\
\text { anxiety state }\end{array}$ & $13.5(13.4-13.6)$ & L20 & $\begin{array}{l}\text { Joint symptom/ } \\
\text { complaint NOS }\end{array}$ & $2.6(2.6-2.7)$ & L86 & $\begin{array}{l}\text { Back syndrome with } \\
\text { radiating pain }\end{array}$ & $7.6(7.5-7.7)$ & $\mathrm{H} 81$ & Excessive ear wax & $1.9(1.9-2.0)$ \\
\hline \multirow[t]{3}{*}{$65-79$} & K86 & $\begin{array}{l}\text { Hypertension } \\
\text { uncomplicated }\end{array}$ & $60.3(60.0-60.6)$ & R74 & $\begin{array}{l}\text { Upper respiratory } \\
\text { infection acute }\end{array}$ & $7.2(7.1-7.3)$ & K86 & $\begin{array}{l}\text { Hypertension } \\
\text { uncomplicated }\end{array}$ & $56.2(55.9-56.5)$ & R74 & $\begin{array}{l}\text { Upper respiratory } \\
\text { infection acute }\end{array}$ & $6.4(6.2-6.5)$ \\
\hline & T93 & Lipid disorder & $52.4(52.1-52.7)$ & U71 & $\begin{array}{l}\text { Cystitis/urinary } \\
\text { infection other }\end{array}$ & $4.3(4.2-4.4)$ & T93 & Lipid disorder & $44.6(44.3-44.9)$ & H81 & Excessive ear wax & $3.9(3.8-4.0)$ \\
\hline & T82 & Obesity & $24.9(24.7-25.1)$ & R78 & $\begin{array}{l}\text { Acute bronchitis/ } \\
\text { bronchiolitis }\end{array}$ & $3.8(3.7-3.9)$ & Y85 & $\begin{array}{l}\text { Benign prostatic } \\
\text { hypertrophy }\end{array}$ & $28.4(28.1-28.7)$ & R78 & $\begin{array}{l}\text { Acute bronchitis/ } \\
\text { bronchiolitis }\end{array}$ & $3.5(3.4-3.6)$ \\
\hline
\end{tabular}


Table 1 Five highest cumulative incidence of acute and prevalence of chronic diseases by sex and age groups (Continued)

\begin{tabular}{|c|c|c|c|c|c|c|c|c|c|c|c|c|}
\hline & L95 & Osteoporosis & $22.8(22.5-23.0)$ & H81 & Excessive ear wax & $3.0(2.9-3.1)$ & T90 & $\begin{array}{l}\text { Diabetes non-insulin } \\
\text { dependent }\end{array}$ & $25.6(25.4-25.9)$ & D82 & Teeth/gum disease & $2.4(2.3-2.5)$ \\
\hline & P76 & Depressive disorder & $22.3(22.1-22.5)$ & L03 & $\begin{array}{l}\text { Low back symptom/ } \\
\text { complaint }\end{array}$ & $2.8(2.7-2.9)$ & T82 & Obesity & $15.4(15.2-15.6)$ & L03 & $\begin{array}{l}\text { Low back symptom/ } \\
\text { complaint }\end{array}$ & $2.3(2.2-2.4)$ \\
\hline \multirow[t]{5}{*}{$80+$} & K86 & $\begin{array}{l}\text { Hypertension } \\
\text { uncomplicated }\end{array}$ & 73.1 (72.7-73.4) & R74 & $\begin{array}{l}\text { Upper respiratory } \\
\text { infection acute }\end{array}$ & $5.1(4.9-5.2)$ & K86 & $\begin{array}{l}\text { Hypertension } \\
\text { uncomplicated }\end{array}$ & $63.4(62.9-63.9)$ & $\mathrm{H} 81$ & Excessive ear wax & $5.8(5.6-6.1)$ \\
\hline & T93 & Lipid disorder & $44.5(44.1-44.9)$ & U71 & $\begin{array}{l}\text { Cystitis/urinary } \\
\text { infection other }\end{array}$ & $5.0(4.9-5.2)$ & Y85 & $\begin{array}{l}\text { Benign prostatic } \\
\text { hypertrophy }\end{array}$ & $37.3(36.8-37.8)$ & R74 & $\begin{array}{l}\text { Upper respiratory } \\
\text { infection acute }\end{array}$ & $5.4(5.1-5.6)$ \\
\hline & L91 & Osteoarthrosis other & $25.7(25.4-26.1)$ & R78 & $\begin{array}{l}\text { Acute bronchitis/ } \\
\text { bronchiolitis }\end{array}$ & $4.5(4.4-4.7)$ & T93 & Lipid disorder & $35.0(34.5-35.5)$ & R78 & $\begin{array}{l}\text { Acute bronchitis/ } \\
\text { bronchiolitis }\end{array}$ & $4.9(4.7-5.2)$ \\
\hline & F92 & Cataract & $23.5(23.2-23.8)$ & H81 & Excessive ear wax & $4.2(4.0-4.4)$ & T90 & $\begin{array}{l}\text { Diabetes non-insulin } \\
\text { dependent }\end{array}$ & $25.4(24.9-25.9)$ & S18 & Laceration/cut & $3.8(3.6-4.0)$ \\
\hline & T90 & $\begin{array}{l}\text { Diabetes non- } \\
\text { insulin dependent }\end{array}$ & $22.8(22.5-23.1)$ & $\mathrm{S} 18$ & Laceration/cut & $3.4(3.3-3.6)$ & F92 & Cataract & $21.9(21.4-22.3)$ & U71 & $\begin{array}{l}\text { Cystitis/urinary } \\
\text { infection other }\end{array}$ & $2.7(2.6-2.9)$ \\
\hline
\end{tabular}

Abbreviations: ICPC 2 International Classification of Primary Care, Cl Confidence interval. 
Table 2 Multimorbidity prevalence and acute diseases, area of residency and visits according to multimorbidity status stratified by sex and age groups

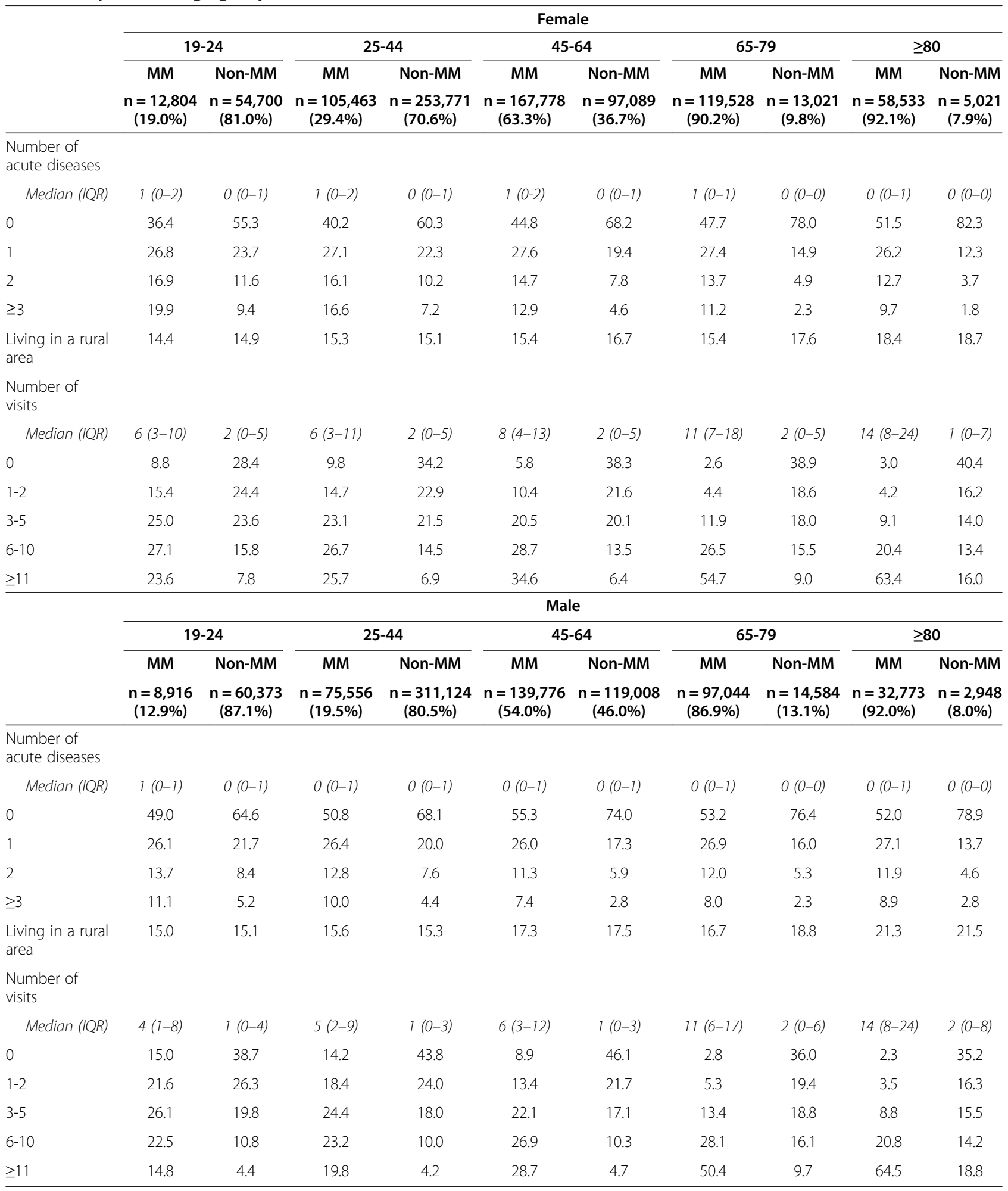

Abbreviations: MM multimorbidity, IQR interquartile range. Data are expressed as percentage, unless otherwise stated. 


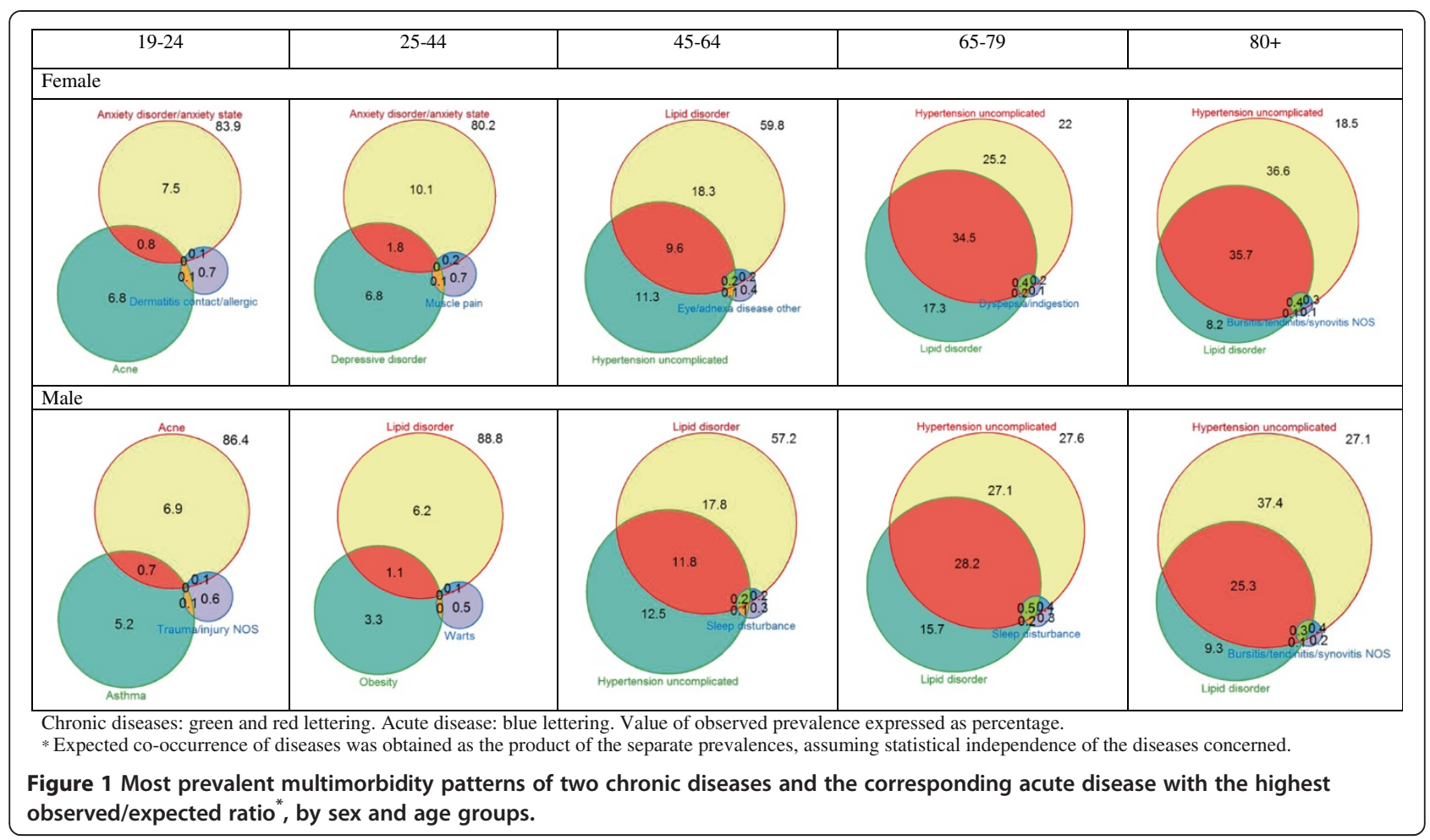

groups. The most frequent multimorbidity pattern of chronic diseases was the combination of hypertension and dyslipidemia in adults over 45 years of age.

We observed a decrease in the number of acute diseases recorded as age increased. Nonetheless, in adjusted models female older than 65 who had acute diseases were more likely to have multimorbidity.

Finally, the use of health services was positively associated with a diagnosis of multimorbidity. Living in a rural area decreased the probability of multimorbidity.

\section{Strengths and weaknesses of the study}

A major strength of this study is the analysis of a large, high-quality database of primary-care records, representative of a large population. In the context of a national health system with universal coverage, EHR data have been shown to yield more reliable and representative conclusions than those derived from survey-based studies [16]. Another important strength was the inclusion of all chronic and acute diagnoses registered in EHR, which contributed to a more accurate analysis of the association between acute and chronic diseases and of the disease combinations present in multimorbidity in this population. To synthesize the results, we present here only the most frequent combinations. Finally, few studies have incorporated acute diseases in the study of multimorbidity patterns [8] and none analyzed the relationship between multimorbidity and acute morbidity.
Some possible biases could have influenced our results. First, diseases could be underreported, especially for male of normal workforce age who tend to see their doctors less often than other strata of patients. This effect would diminish in the two oldest age groups because of the retirement age (65 years) in Spain. In patients with multimorbidity, the true incidence of acute disease could be underreported because the GP would place higher priority on the chronic problems in these patients. On the other hand, there could be an over-representation of chronic diagnoses (e.g., hypertension, diabetes, hyperlipidemia, etc.) that are included in the goals/incentives contracts of Catalan PHCT.

The diseases that form part of the CatSalut treatment objectives may be more carefully recorded than other conditions. However, these same diseases are the most prevalent (high blood pressure, diabetes, hypercholesterolemia, smoking, dyslipidemia, ischemic heart disease, atrial fibrillation) and therefore have the greatest impact on population health. The quality-recorders database (SIDIAP-Q) used in this study minimizes the under-reporting of diseases not included in the CatSalut objectives.

Furthermore, the stratified analysis allows more accurate estimation within each age-sex strata and universal access to free health care and medications makes it more likely that patients seeking care will acquire a diagnosis, either acute or chronic $[17,18]$. Second, there is no universally accepted criterion for consensus classification of acute and chronic disease. This lack of accurate case definitions impedes the establishment of the true incidence/prevalence 
Table 3 Cumulative incidence and risk ratio (RR) of multimorbidity for the five acute diseases with the highest cumulative incidence by sex and age groups* ${ }^{*}$

\begin{tabular}{|c|c|c|c|c|c|c|c|c|c|c|c|c|}
\hline \multicolumn{7}{|c|}{ Female } & \multicolumn{6}{|c|}{ Male } \\
\hline \multirow[t]{2}{*}{$\begin{array}{l}\text { Age } \\
\text { groups }\end{array}$} & \multirow[t]{2}{*}{$\begin{array}{l}\text { ICPC } 2 \\
\text { code }\end{array}$} & \multirow[t]{2}{*}{ Acute diseases } & \multirow{2}{*}{$\begin{array}{c}\mathrm{MM} \\
\mathrm{Cl} \\
(\%)\end{array}$} & \multirow{2}{*}{$\begin{array}{c}\text { Non-MM } \\
\mathrm{Cl} \\
(\%)\end{array}$} & \multirow[t]{2}{*}{ Risk ratio } & \multirow[t]{2}{*}{$\begin{array}{l}95 \% \text { confidence } \\
\text { interval }\end{array}$} & \multirow[t]{2}{*}{$\begin{array}{l}\text { ICPC } 2 \\
\text { code }\end{array}$} & \multirow[t]{2}{*}{ Acute diseases } & \multirow{2}{*}{$\begin{array}{l}\mathrm{MM} \\
\mathrm{Cl}^{*} \\
(\%)\end{array}$} & \multirow{2}{*}{$\begin{array}{c}\text { Non-MM } \\
\mathrm{Cl} \\
(\%)\end{array}$} & \multirow[t]{2}{*}{ Risk ratio } & \multirow[t]{2}{*}{$\begin{array}{l}95 \% \text { confidence } \\
\text { interval }\end{array}$} \\
\hline & & & & & & & & & & & & \\
\hline \multirow[t]{5}{*}{$19-24$} & R74 & Upper respiratory infection, acute & 16.2 & 8.8 & 1.10 & $(1.04-1.16)$ & R74 & Upper respiratory infection, acute & 11.9 & 7.0 & 0.96 & $(0.89-1.03)$ \\
\hline & R76 & Tonsillitis, acute & 6.7 & 4.3 & 0.94 & $(0.86-1.02)$ & R76 & Tonsillitis, acute & 5.5 & 3.4 & 0.92 & $(0.83-1.02)$ \\
\hline & U71 & Cystitis/urinary infection, other & 6.3 & 4.1 & 0.85 & $(0.78-0.93)$ & D73 & Gastroenteritis, presumed infection & 4.8 & 2.8 & 0.93 & $(0.83-1.04)$ \\
\hline & D82 & Teeth/gum disease & 6.2 & 3.6 & 1.14 & $(1.04-1.25)$ & D82 & Teeth/gum disease & 4.3 & 2.7 & 0.63 & $(0.44-0.89)$ \\
\hline & D73 & Gastroenteritis, presumed infection & 6.3 & 3.4 & 1.06 & $(0.97-1.16)$ & S16 & Bruise/contusion & 3.2 & 2.4 & 0.75 & $(0.66-0.86)$ \\
\hline \multirow[t]{5}{*}{$25-44$} & R74 & Upper respiratory infection, acute & 12.8 & 6.9 & 1.01 & $(0.98-1.03)$ & R74 & Upper respiratory infection, acute & 9.9 & 5.7 & 0.85 & $(0.82-0.87)$ \\
\hline & L03 & Low back symptom/complaint & 4.4 & 3.1 & 0.73 & $(0.71-0.76)$ & D73 & Gastroenteritis, presumed infection & 3.8 & 2.3 & 0.79 & $(0.76-0.83)$ \\
\hline & U71 & Cystitis/urinary infection other & 4.4 & 2.6 & 0.86 & $(0.83-0.90)$ & L03 & Low back symptom/complaint & 3.5 & 2.2 & 0.75 & $(0.71-0.78)$ \\
\hline & D73 & Gastroenteritis presumed infection & 4.3 & 2.5 & 0.90 & $(0.87-0.94)$ & D82 & Teeth/gum disease & 3.5 & 2.2 & 0.82 & $(0.72-0.93)$ \\
\hline & R76 & Tonsillitis, acute & 3.6 & 2.5 & 1.08 & $(0.91-1.29)$ & R76 & Tonsillitis, acute & 2.4 & 1.8 & 0.69 & $(0.65-0.73)$ \\
\hline \multirow[t]{5}{*}{$45-64$} & R74 & Upper respiratory infection, acute & 9.7 & 4.5 & 0.92 & $(0.88-0.95)$ & R74 & Upper respiratory infection, acute & 6.7 & 3.4 & 0.79 & $(0.76-0.82)$ \\
\hline & L03 & Low back symptom/complaint & 3.9 & 2.1 & 0.78 & $(0.74-0.82)$ & L03 & Low back symptom/complaint & 3.2 & 2.1 & 0.58 & $(0.55-0.62)$ \\
\hline & U71 & Cystitis/urinary infection, other & 4.0 & 1.7 & 0.86 & $(0.81-0.92)$ & R78 & Acute bronchitis/bronchiolitis & 3.0 & 1.3 & 0.86 & $(0.80-0.92)$ \\
\hline & R78 & Acute bronchitis/bronchiolitis & 3.9 & 1.6 & 0.95 & $(0.89-1.01)$ & D82 & Teeth/gum disease & 2.9 & 1.6 & 0.83 & $(0.70-0.98)$ \\
\hline & L20 & Joint symptom/complaint NOS & 3.3 & 1.5 & 0.97 & $(0.82-1.15)$ & $\mathrm{H} 81$ & Excessive ear wax & 2.5 & 1.4 & 0.74 & $(0.69-0.78)$ \\
\hline \multirow[t]{5}{*}{ 65-79 } & R74 & Upper respiratory infection, acute & 8.4 & 3.2 & 0.92 & $(0.70-1.20)$ & R74 & Upper respiratory infection, acute & 7.6 & 3.3 & 0.93 & $(0.84-1.03)$ \\
\hline & U71 & Cystitis/urinary infection other & 5.1 & 1.4 & 1.09 & $(0.91-1.30)$ & $\mathrm{H} 81$ & Excessive ear wax & 4.4 & 2.3 & 0.74 & $(0.65-0.83)$ \\
\hline & R78 & Acute bronchitis/bronchiolitis & 4.5 & 1.3 & 0.71 & $(0.36-1.39)$ & R78 & Acute bronchitis/bronchiolitis & 4.2 & 1.5 & 0.99 & $(0.85-1.14)$ \\
\hline & $\mathrm{H} 81$ & Excessive ear wax & 3.3 & 1.2 & 0.88 & $(0.74-1.04)$ & D82 & Teeth/gum disease & 2.9 & 1.2 & 1.31 & $(1.10-1.57)$ \\
\hline & L03 & Low back symptom/complaint & 3.0 & 1.1 & 0.76 & $(0.54-1.07)$ & L03 & Low back symptom/complaint & 2.5 & 1.1 & 0.87 & $(0.73-1.03)$ \\
\hline \multirow[t]{5}{*}{$80+$} & R74 & Upper respiratory infection, acute & 5.8 & 1.5 & 1.47 & $(1.16-1.86)$ & $\mathrm{H} 81$ & Excessive ear wax & 6.5 & 2.7 & 0.98 & $(0.77-1.24)$ \\
\hline & U71 & Cystitis/urinary infection, other & 5.9 & 1.5 & 1.40 & $(1.10-1.78)$ & R74 & Upper respiratory infection, acute & 6.2 & 2.0 & 1.29 & $(0.98-1.70)$ \\
\hline & R78 & Acute bronchitis/bronchiolitis & 5.3 & 2.0 & 0.99 & $(0.80-1.23)$ & R78 & Acute bronchitis/bronchiolitis & 5.7 & 2.0 & 1.06 & $(0.80-1.40)$ \\
\hline & H81 & Excessive ear wax & 4.7 & 1.3 & 1.34 & $(1.04-1.73)$ & S18 & Laceration/cut & 4.3 & 2 & 0.75 & $(0.57-0.99)$ \\
\hline & S18 & Laceration/cut & 3.9 & 1.6 & 0.78 & $(0.61-0.99)$ & U71 & Cystitis/urinary infection, other & 3.0 & 1.5 & 0.73 & $(0.53-1.01)$ \\
\hline
\end{tabular}

Abbreviations: MM multimorbidity, RR risk ratio, ICPC 2 International Classification of Primary Care, Cl Cumulative incidence, NOS not otherwise specified.
${ }^{*}$ Cumulative incicidence was calculated as the sum of the number of new acute events during the study period divided by the 2010 population (e.g. if a patient passed two bronchitis in one year. the total number of events accounted for was two).

"The outcome is the number of acute diseases in each person for each acute disease considered. RR of multimorbidity versus non-multimorbidity was calculated by Poisson, negative binomial (if overdispersion is present) or zero inflated (when data showed excess of zero counts) equation as appropriate and adjusted for number of visits and area of residency. In bold $\mathrm{P}<0.05$. 
Table 4 Factors associated with multimorbidity by sex and age groups

\begin{tabular}{|c|c|c|c|c|c|c|c|c|c|c|c|c|c|c|c|}
\hline \multicolumn{16}{|c|}{ Female } \\
\hline & \multicolumn{3}{|c|}{$19-24$} & \multicolumn{3}{|c|}{$25-44$} & \multicolumn{3}{|c|}{$45-64$} & \multicolumn{3}{|c|}{$65-79$} & \multicolumn{3}{|c|}{$80+$} \\
\hline & OR & $95 \% \mathrm{Cl}$ & P-value & OR & $95 \% \mathrm{Cl}$ & P-value & OR & $95 \% \mathrm{Cl}$ & P-value & OR & $95 \% \mathrm{Cl}$ & P-value & OR & $95 \% \mathrm{Cl}$ & P-value \\
\hline Number of acute diseases(ref.0) & & & 0.030 & & & $<0.001$ & & & $<0.001$ & & & $<0.001$ & & & $<0.001$ \\
\hline 1 & 0.94 & 0.89-0.99 & & 0.88 & $0.86-0.90$ & & 0.82 & $0.80-0.84$ & & 0.98 & $0.92-1.03$ & & 1.16 & $1.05-1.28$ & \\
\hline 2 & 0.95 & $0.89-1.01$ & & 0.86 & $0.83-0.88$ & & 0.76 & $0.73-0.78$ & & 1.05 & $0.96-1.15$ & & 1.54 & $1.31-1.80$ & \\
\hline$\geq 3$ & 1.02 & $0.95-1.09$ & & 0.89 & $0.87-0.92$ & & 0.78 & $0.75-0.81$ & & 1.29 & $1.14-1.46$ & & 1.99 & $1.59-2.47$ & \\
\hline Rural area (ref. urban) & 0.94 & 0.89-0.99 & 0.025 & 0.94 & $0.92-0.96$ & $<0.001$ & 0.80 & $0.78-0.82$ & $<0.001$ & 0.69 & $0.65-0.73$ & $<0.001$ & 0.76 & $0.70-0.83$ & $<0.001$ \\
\hline Number of visits (ref. 0) & & & $<0.001$ & & & $<0.001$ & & & $<0.001$ & & & $<0.001$ & & & $<0.001$ \\
\hline $1-2$ & 2.09 & $1.93-2.26$ & & 2.34 & $2.28-2.14$ & & 3.40 & $3.30-3.51$ & & 3.62 & $3.38-3.87$ & & 3.41 & $3.08-3.78$ & \\
\hline $3-5$ & 3.52 & $3.25-3.80$ & & 4.04 & $3.93-4.15$ & & 7.54 & $7.31-7.77$ & & 10.18 & $9.54-10.86$ & & 8.36 & $7.55-9.27$ & \\
\hline $6-10$ & 5.69 & $5.25-6.17$ & & 7.11 & $6.91-7.32$ & & 16.28 & $15.76-16.81$ & & 25.98 & $24.28-27.80$ & & 18.86 & $16.99-20.94$ & \\
\hline$\geq 11$ & 9.93 & $9.09-10.85$ & & 14.52 & $14.05-14.99$ & & 43.03 & $41.39-44.73$ & & 90.81 & $83.74-98.48$ & & 45.19 & $40.69-50.19$ & \\
\hline \multicolumn{16}{|c|}{ Male } \\
\hline & \multicolumn{3}{|l|}{$19-24$} & \multicolumn{3}{|l|}{$25-44$} & \multicolumn{3}{|l|}{$45-64$} & \multicolumn{3}{|l|}{$65-79$} & \multicolumn{3}{|l|}{$80+$} \\
\hline & OR & $95 \% \mathrm{Cl}$ & P-value & OR & $95 \% \mathrm{Cl}$ & P-value & OR & $95 \% \mathrm{Cl}$ & P-value & OR & $95 \% \mathrm{Cl}$ & P-value & OR & $95 \% \mathrm{Cl}$ & P-value \\
\hline Number of acute diseases(ref.0) & & & $<0.001$ & & & $<0.001$ & & & $<0.001$ & & & $<0.001$ & & & 0.590 \\
\hline 1 & 0.78 & $0.73-0.83$ & & 0.71 & $0.70-0.73$ & & 0.67 & $0.65-0.69$ & & 0.84 & $0.80-0.89$ & & 1.07 & $0.95-1.21$ & \\
\hline 2 & 0.79 & $0.73-0.86$ & & 0.64 & $0.62-0.66$ & & 0.58 & $0.56-0.60$ & & 0.78 & $0.72-0.85$ & & 1.08 & $0.89-1.31$ & \\
\hline$\geq 3$ & 0.76 & $0.69-0.83$ & & 0.60 & $0.58-0.62$ & & 0.55 & $0.52-0.57$ & & 0.86 & $0.77-0.97$ & & 1.12 & $0.88-1.42$ & \\
\hline Rural area (ref. urban) & 0.94 & $0.88-1.00$ & 0.045 & 0.95 & $0.93-0.97$ & $<0.001$ & 0.87 & $0.85-0.89$ & $<0.001$ & 0.71 & $0.68-0.75$ & $<0.001$ & 0.79 & $0.71-0.88$ & $<0.001$ \\
\hline Number of visits (ref. 0) & & & $<0.001$ & & & $<0.001$ & & & $<0.001$ & & & $<0.001$ & & & $<0.001$ \\
\hline $1-2$ & 2.32 & $2.15-2.50$ & & 2.67 & $2.60-2.75$ & & 3.57 & $3.47-3.67$ & & 3.58 & $3.35-3.82$ & & 3.33 & $2.89-3.85$ & \\
\hline $3-5$ & 3.93 & $3.64-4.25$ & & 5.18 & $5.04-5.33$ & & 8.20 & $7.97-8.43$ & & 9.66 & $9.06-10.30$ & & 8.62 & $7.50-9.92$ & \\
\hline $6-10$ & 6.51 & $5.97-7.09$ & & 9.62 & $9.33-9.92$ & & 17.78 & $17.24-18.34$ & & 24.51 & $22.93-26.19$ & & 22.07 & $19.13-25.47$ & \\
\hline$\geq 11$ & 10.86 & $9.82-12.01$ & & 20.58 & $19.86-21.32$ & & 45.13 & $43.43-46.90$ & & 76.41 & $70.68-82.60$ & & 51.32 & 44.49-59.19 & \\
\hline
\end{tabular}

Abbreviations: OR odds ratio, $\mathrm{Cl}$ confidence interval, ref. reference. 
of a disease [19]. Finally, a residual confounding cannot be completely excluded, and could occur because of epidemiological factors not considered in this study, such as patients' socioeconomic status [20].

\section{Strengths and weaknesses in relation to other studies}

The estimated multimorbidity prevalence in our sample is higher than in other European studies [21-24], perhaps because of the analysis of a greater number of diseases in our study than in most other published studies [25]. Nonetheless, the patterns of multimorbidity observed were similar to those observed in other studies [26].

As in other studies, multimorbidity was more prevalent among female $[21,22,24]$. This could be due to the longer female life expectancy and worse health status, compared to male, differences that are due to both biological and social factors [26]. In addition, sex is a social determinant that influences health status, health behaviours and the use of health services [27-30]. Recent studies, however, suggest a dismantling of this paradigm based on sex-stratified analysis of consultations for common symptoms [31].

Acute problems are time-consuming for health professional [32], and therefore should be considered part of the primary care workload. Although current health policy, health care services, and research are all heavily focused on chronic diseases, we must not forget that $41 \%$ of primary care visits are motivated by an acute disease [33]. The incidence of acute diseases observed in our study concurs with other reports of acute upper respiratory infection and other health problems of infectious aetiology (acute tonsillitis, cystitis) as the primary reasons for seeking primary care, along with non-infectious diseases such as dorsalgia [33,34].

Our study observed a higher prevalence of multimorbidity in rural settings. Other studies conducted in rural areas have reported only a greater prevalence of multimorbidity in elderly people [6,7]. Nonetheless, our adjusted analysis showed that living in a rural area is negatively associated with multimorbidity. This phenomenon could be due to the environmental and sociocultural context and access to both public and private services [28].

\section{Implications for clinicians and policymakers}

Our study considered multimorbidity in patients who received primary medical care, considering all visits and diagnoses (acute and chronic diseases). This approach allowed the identification of vulnerable subgroups in our population. A major advantage of our methodology is the use of data obtained directly from standard clinical practice. Knowing the distribution of acute and chronic diseases by life-stage and sex will help the clinician faced with a particular patient to anticipate disease patterns based on the patient's sex and stage of life, recognizing that these vary with age. This will encourage the implementation of personalized disease prevention and health promotion activities.

At the level of health policy and health care administration, the organization of services should be reviewed to ensure that continuity and coordination of patient care are guaranteed; current evidence suggests the potential for improvement in this regard [35].

\section{Unanswered questions and future research}

The classification of chronic and acute disease remains unresolved, and there is no consensus on the type and number of chronic diseases that define multimorbidity. A personalized measure to determine the severity of diagnosed multimorbidity is also needed.

If longitudinal studies confirm a higher incidence of morbidity in patients with multimorbidity, evidence-based interventions will be needed to prevent the onset of acute disease. Further studies are needed to study possible genetic and pathophysiological explanations that corroborate the observed multimorbidity patterns.

In-depth analysis of other contextual factors related to multimorbidity is also required, along with studies of the relationship between area of residence and multimorbidity and of the differences in health status that may exist between different territories. Finally, there is a need for the implementation and evaluation of health literacy and selfmanagement interventions to improve patient competence in resolving routine acute diseases, which in turn will decrease the care burden in primary care systems.

\section{Conclusions}

Multimorbidity is related to more use of health services and higher incidence of acute diseases, which increases the burden on primary care services. Residence in urban vs rural settings is a factor for future in-depth study.

The association of acute morbidity, area of residency or use of health services with multimorbidity differs according to life-stage and sex. Therefore, the study of multimorbidity should be stratified by life-stage and sex. Understanding these trends across life-stages will allow health systems to adjust their clinical and management models to adapt and prioritize interventions.

\footnotetext{
Abbreviations

PHCT: Primary health care teams; CHI: Catalan Health Institute; EHR: Electronic health records; SIDIAP: Information System for the Development of Research in Primary Care; GP: General practitioners; ICD: International Classification of Diseases; ICPC: International Classification of Primary Care; IDIAP: Institut Universitari d'Investigació en Atenció Primària; SD: Standard deviation; IQR: Interquartile range; RR: Risk ratios.
}

\section{Competing interests}

The authors declare that they have no competing interests.

\section{Authors' contributions}

All authors contributed to the design of the study, revised the article, and approved the final version. CV, QFB, JMV, MMP, ARL drafted the study protocol and obtained the funding. TRB, CV, QFB, JMV, ARL contributed to 
the analysis and interpretation of data. CV, QFB, JMV, TRB, ARL, MPV, MMP, and EPR wrote the first draft, and all authors contributed ideas, interpreted the findings and reviewed rough drafts of the manuscript. All authors read and approved the final manuscript.

\section{Acknowledgments}

We thank the Catalan Health Institute and especially the SIDIAP Unit, which provided the database for the study. The authors also appreciate the English language review by Elaine Lilly, PhD.

\section{Funding}

This study was funded by the Ministry of Science and Innovation through the Instituto Carlos III (ISCiii) as part of the Primary Care Prevention and Health Promotion Research Network (rediAPP), by ISCiii-RETICS (RD12/0005), by a grant for research projects ISCiii (PI12/00427), and by a 2011-2013 scholarship that aims to promote research in Primary Health Care by health professionals who have completed their specialty training, awarded by Institut Universitari d'Investigació en Atenció Primària Jordi Gol (IDIAP Jordi Gol). The funders had no role in the study design, collection, analysis and interpretation of data, writing of the manuscript and decision to submit for publication.

\section{Author details}

'Institut Universitari d'Investigació en Atenció Primària Jordi Gol (IDIAP Jord Gol), Gran Via Corts Catalanes, 587 àtic, 08007 Barcelona, Spain. ${ }^{2}$ Universitat Autònoma de Barcelona, Plaza Cívica, Campus de la UAB, 08193 Bellaterra, Cerdanyola del Vallès, Spain. ${ }^{3}$ Hospital de Campdevànol, Ctra. de Gombrèn, 20, 17530 Campdevànol, Spain. ${ }^{4}$ Health Services \& Policy Research Group, School of Medicine, University of Exeter, Exeter EX1 2LU, UK.

Received: 31 December 2013 Accepted: 21 March 2014 Published: 26 March 2014

\section{References}

1. Bodenheimer T, Wagner EH, Grumbach K: Improving primary care for patients with chronic illness. JAMA 2002, 288:1775-1779.

2. Van den Akker M, Buntinx F, Knottnerus JA: Comorbidity or multimorbidity: what's in a name? A review of literature. Eur J Gen Pract 1996, 2:65-70.

3. Freund T, Kunz CU, Ose D, Szecsenyi J, Peters-Klimm F: Patterns of multimorbidity in primary care patients at high risk of future hospitalization. Popul Health Manag 2012, 15:119-124.

4. Gijsen R, Hoeymans N, Schellevis FG, Ruwaard D, Satariano WA, van den Bos GA: Causes and consequences of comorbidity: a review. J Clin Epidemiol 2001, 54:661-674.

5. van den Akker M, Buntinx F, Roos S, Knottnerus JA: Problems in determining occurrence rates of multimorbidity. J Clin Epidemiol 2001, 54:675-679.

6. Khanam MA, Streatfield PK, Kabir ZN, Qiu C, Cornelius C, Wahlin A: Prevalence and patterns of multimorbidity among elderly people in rura Bangladesh: a cross-sectional study. J Health Popul Nutr 2011, 29:406-414

7. John R, Kerby DS, Hennessy CH: Patterns and impact of comorbidity and multimorbidity among community-resident American Indian elders. Gerontologist 2003, 43:649-660

8. Saltman DC, Sayer GP, Whicker SD: Co-morbidity in general practice. Postgrad Med J 2005, 81:474-480.

9. Broemeling A, Watson D, Black C: Chronic conditions. Co-morbidity among residents of British Columbia. Vancouver, BC: University of British Columbia; 2005.

10. World Health Organization: A Glossary of Terms for Community Health Care and Services for Older Persons. Kobe: WHO; 2004.

11. Wolff JL, Starfield B, Anderson G: Prevalence, expenditures, and complications of multiple chronic conditions in the elderly. Arch Intern Med 2002, 162:2269-2276.

12. Information system for the development of research in primary care (SIDIAP data base). [http://www.sidiap.org/]

13. García-Gil Mdel M, Hermosilla E, Prieto-Alhambra D, Fina F, Rosell M, Ramos R, Rodriguez J, Williams T, Van Staa T, Bolibar B: Construction and validation of a scoring system for the selection of high-quality data in a Spanish population primary care database (SIDIAP). Inform Prim Care 2011, 19(3):135-145.

14. O'Halloran J, Miller GC, Britt H: Defining chronic conditions for primary care with ICPC-2. Fam Pract 2004, 21:381-386

15. Defining Chronic Conditions for Primary Care Using ICPC-2. Available in: http://www.fmrc.org.au/Download/DefiningChronicConditions.pdf.
16. Violán C, Foguet-Boreu Q, Hermosilla-Pérez E, Valderas JM, Bolíbar B, FàbregasEscurriola M, Brugulat-Guiteras P, Muñoz-Pérez MÁ: Comparison of the information provided by electronic health records data and a population health survey to estimate prevalence of selected health conditions and multimorbidity. BMC Public Health 2013, 13:251

17. Jordan K, Porcheret M, Croft P: Quality of morbidity coding in general practice computerized medical records: a systematic review. Fam Pract 2004, 21:396-412.

18. Valderas JM, Starfield B, Sibbald B, Salisbury C, Roland M: Defining comorbidity: implications for understanding health and health services. Ann Fam Med 2009, 7:357-363.

19. de Lusignan S, Tomson C, Harris K, van Vlymen J, Gallagher H: Creatinine fluctuation has a greater effect than the formula to estimate glomerular filtration rate on the prevalence of chronic kidney disease. Nephron Clin Pract 2011, 117:c213-c224.

20. Barnett K, Mercer SW, Norbury M, Watt G, Wyke S, Guthrie B: Epidemiology of multimorbidity and implications for health care, research, and medical education: a cros-sectional study. Lancet 2012, 380:37-43.

21. Prados-Torres A, Poblador-Plou B, Calderón-Larrañaga A, Gimeno-Feliu LA, González-Rubio F, Poncel-Falcó A, Sicras-Mainar A, Alcalá-Nalvaiz JT: Multimorbidity patterns in primary care: interactions among chronic diseases using factor analysis. PLoS One 2012, 7:e32190.

22. Glynn LG, Valderas JM, Healy P, Burke E, Newell J, Gillespie P, Murphy AW: The prevalence of multimorbidity in primary care and its effect on health care utilization and cost. Fam Pract 2011, 28:516-523.

23. Salisbury C, Johnson L, Purdy S, Valderas JM, Montgomery AA Epidemiology and impact of multimorbidity in primary care: a retrospective cohort study. Br J Gen Pract 2011, 61:e12-e21.

24. García-Olmos L, Salvador CH, Alberquilla Á, Lora D, Carmona M, GarcíaSagredo P, Pascual M, Muñoz A, Monteagudo JL, García-López F: Comorbidity patterns in patients with chronic diseases in general practice. PLoS One 2012, 7:e32141.

25. Marengoni A, Angleman S, Melis R, Mangialasche F, Karp A, Garmen A, Meinow B, Fratiglioni L: Aging with multimorbidity: a systematic review of the literature. Ageing Res Rev 2011, 10:430-439.

26. Laux G, Kuehlein T, Rosemann T, Szecsenyi J: Co- and multimorbidity patterns in primary care based on episodes of care: results from the German CONTENT project. BMC Health Serv Res 2008, 8:14.

27. Lee JH, Sadana R: Improving Equity in Health by Addressing Social Determinants. The Commission on Social Determinants of Health Knowledge Networks. World Health Organization; 2011 [http://whqlibdoc.who.int/ publications/2011/9789241503037_eng.pdf]

28. Marmot M, Friel S, Bell R, Houweling TA, Taylor S: Commission on social determinants of health: closing the gap in a generation: health equity through action on the social determinants of health. Lancet 2008, 372:1661-1669.

29. Bertakis KD, Azari R, Helms LJ, Callahan EJ, Robbins JA: Gender differences in the utilization of health care services. J Fam Pract 2000, 49:147-152.

30. Redondo-Sendino A, Guallar-Castillón P, Banegas JR, Rodríguez-Artalejo F: Gender differences in the utilization of health-care services among the older adult population of Spain. BMC Public Health 2006, 6:155.

31. Hunt K, Adamson J, Hewitt C, Nazareth J: Do women consult more than men? A review of gender and consultation for back pain and headache $J$ Health Serv Res Policy 2011, 16:108-117.

32. Stange KC, Zyzanski SJ, Jaén CR, Callahan EJ, Kelly RB, Gillanders WR, Shank JC, Chao J, Medalie JH, Miller WL, Crabtree BF, Flocke SA, Gilchrist VJ, Langa DM, Goodwin MA: Illuminating the 'black box'. A description of 4454 patient visits to 138 family physicians. J Fam Pract 1998, 46:377-389.

33. Woodwell DA, Cherry DK: National ambulatory medical care survey: 2002 summary. Adv Data 2004, 346:1-44

34. Wändell $P$, Carlsson AC, Wettermark B, Lord G, Cars T, Ljunggren G: Most common diseases diagnosed in primary care in Stockholm, Sweden, in 2011. Fam Pract 2013, 506:13

35. Smith SM, Soubhi H, Fortin M, Hudon C, O'Dowd T: Managing patients with multimorbidity: systematic review of interventions in primary care and community settings. BMJ 2012, 345:e5205.

doi:10.1186/1471-2296-15-55

Cite this article as: Foguet-Boreu et al: Impact of multimorbidity: acute morbidity, area of residency and use of health services across the life span in a region of south Europe. BMC Family Practice 2014 15:55. 